\title{
Il punto su epatite $B$ e $C$ in dialisi: riflessioni sulla contumacia dei pazienti
}

\author{
Marco Lombardi
}

S.C. Nefrologia e Dialisi Ospedale S.M. Annunziata e S.S. Dialisi del Mugello, Azienda Sanitaria di Firenze, Firenze

MAKING THE POINT ON HePATITIS B ANd C In Dialysis: ROLE OF PATIENTS' ISOlation

Abstract. The present work tries to analyze the role of isolation in relation to the risks of infections' transmission in hospitalized hemodialysis patients. To better explain these issues, the author compares two articles published on the Italian Journal of Nephrology. The first is based on data and protocols issued by the main agency for the prevention of infections (the CDC) together with the well-known Universal and Special Precautions, suggesting supplementary measures such as the isolation of HBV-positive patients.

The second paper states that the best precautionary measure to prevent infections' spreading is to constantly use all Standard and Special precautions, together with a continuous monitoring of the measures adopted and their results.

Another criticism moved to the measure of patients' isolation is related to the incomplete protection provided by anti-HBs antibodies, as well as to the possible development of hidden infections.

This discussion is very important since, during the actual shortening of economic resources, it could be crucial to avoid patients' isolation in dedicated areas of the hemodialysis units.

If Universal and Special procedures are correctly applied, viruses will not be able to grow on any surface, allowing $\mathrm{HBV}$-positive hemodialysis patients to share the same spaces with the other patients.

Key words: Universal and Special Precautions, Patients' isolation, HBV and HCV, Spreading infections preventing, Hemodialysis

Conflict of interest: None.

Financial support: None.

Accettato: 16 Febbraio 2014

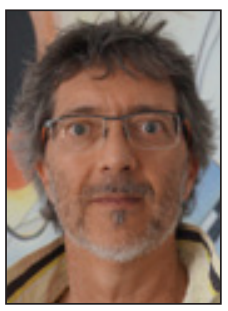

Marco Lombardi

\section{Introduzione}

Il presente contributo, tratto dalla relazione che ho tenuto il 13 Dicembre del 2013 a Cuneo presso il Convegno "Il Punto su Epatite B e C in Dialisi", vuole cercare di analizzare criticamente il ruolo della contumacia in dialisi in relazione al rischio della trasmissione delle infezioni ematogene in emodialisi ospedaliera. Infatti, tale problematica non sussiste nella dialisi domiciliare, rappresentata essenzialmente dalla dialisi peritoneale e, in minor misura, dall'emodialisi domiciliare $(1,2)$.

A tal proposito, mi sono avvalso degli ultimi due articoli apparsi sul Giornale Italiano di Nefrologia (GIN, organo ufficiale della Società Italiana di Nefrologia (SIN)) e di altri reperiti dalla letteratura internazionale sull'argomento epatite $\mathrm{C}$ e $\mathrm{B}$, ovvero sui due patogeni ematogeni più diffusi e meglio conosciuti nell'ambiente nefro-dialitico. In realtà, i patogeni ematogeni più citati, sono, in ordine cronologico della loro conoscenza, HBV, HCV e HIV, ma le riflessioni che seguono sono applicabili e dovrebbero essere applicate a tutti i pato- geni ematogeni, anche a quelli ritenuti meno "pericolosi" o "aggressivi" e a quelli di cui non abbiamo ancora piena conoscenza né consapevolezza.

\section{La visione corrente}

Nel più recente articolo apparso sul GIN nel 2013, Fabrizio Fabrizi, uno dei maggiori esperti italiani sull'argomento, produce una completa revisione sui tre principali patogeni ematogeni virali in emodialisi (3). La sua review si fonda principalmente sui propri dati e su quelli dei CDC (Centers for Disease Control and Prevention) di Atlanta, la maggiore e più qualificata fonte di informazioni e raccomandazioni su questo argomento. Nel suo articolo, Fabrizi ha come scopo quello di ... effettuare un aggiornamento riguardo le raccomandazioni per un 'appropriata prevenzione di HBV, HCV e HIV nei centri dialisi... (3). Il punto di partenza della revisione di Fabrizi è che tali infezioni sono tuttora diffuse nei centri dialitici, anche nel cosiddetto mondo industrializzato, in modo per nulla trascurabile: dati multicentrici (308 centri dialisi per un totale di oltre 8000 pazienti) riportati dal DOPPS (Dialysis Outcomes and Practice Patterns Study) indicano una prevalenza di emodializzati 
HBsAg positivi tra 1 ' $1 \%$ e il $5 \%$ e di antiHCV positivi fra il $3 \%$ e il $23 \%(4,5)$, mentre i CDC statunitensi mostrano prevalenze dell' $1 \%$ per HBsAg e del $7.8 \%$ per antiHCV positività su oltre 263000 pazienti (6).

L'articolo analizza, quindi, tali dati in rapporto al loro divenire e il loro divenire in rapporto alle misure precauzionali assunte, facendosi portatore principalmente di quanto espresso dagli stessi CDC.

Vengono citati studi assai noti anche se datati, a riprova della scarsa letteratura in merito, in cui la riduzione della prevalenza di pazienti HBsAg positivi (7) viene messa in relazione con l'uso degli screening trasfusionali per il virus (indiretti prima e diretti poi), con la riduzione delle pratiche emotrasfusionali (conseguente alla diffusione degli agenti stimolanti la eritropoiesi, ESA), con la vaccinazione antiHBV e, infine, con le procedure di controllo adottate nei centri dialisi. A questo riguardo, sono citate le precauzioni cosiddette Universali o Standard e quelle Specifiche o Speciali per l'emodialisi, a noi note e già descritte nei contributi del GIN che precedono questo articolo. In aggiunta a queste precauzioni, per i pazienti HBsAg positivi, vengono raccomandate, sempre dai CDC, alcune procedure Supplementari (Tab. I).

Pertanto, l'Autore puntualizza che tutti i centri di nuova apertura dovrebbero prevedere sale di isolamento per attuare la contumacia dei pazienti HBsAg positivi, mentre, nei centri già esistenti e sprovvisti di sale contumaciali, $i$ pazienti HBsAg positivi dovrebbero essere tenuti separati da quelli negativi-suscettibili di infezione, cioè trattati in un'area "lontana" da quelle in cui si svolge l'attività quotidiana di maggiore intensità. Viceversa, negli stessi centri sforniti di aree contumaciali, i pazienti positivi e i negativiprotetti (cioè vaccinati e con titolo antiHBsAg protettivo) potrebbero essere dializzati nello stesso turno, nella stessa stanza e sulle stesse macchine e gestiti contemporaneamente dallo stesso personale.

\section{Una visione contro-corrente}

Nel 2009, insieme alla Dr.ssa Chiara Cherubini, abbiamo pubblicato sul GIN, nell'ambito di una serie di articoli sul rischio clinico, una revisione sull'importanza fondamentale che ha la mancata osservazione delle precauzioni Speciali nella trasmissione delle infezioni tra i pazienti emodializzati nei

\section{TABELLA I - RACCOMANDAZIONI SUPPLEMENTARI DEI CDC PER I PAZIENTI HBsAg POSITIVI}

Isolare i pazienti positivi in sale oppure in aree separate

Usare macchine di dialisi dedicate per i pazienti positivi

Non riutilizzare i filtri usati per i pazienti positivi

Lo staff dei centri dialisi non deve seguire contemporaneamente (nello stesso turno o in turni successivi) pazienti positivi e pazienti suscettibili di infezione

Vaccinazione antiHBV dei pazienti negativi e screening mensile per HBsAg per quelli suscettibili di infezione

Lo staff che segue i pazienti positivi deve essere protetto dall'HBV nosocomi (8).

In quell'articolo abbiamo tentato di ribadire che l'adozione di tutte le precauzioni Universali e Speciali è la misura preventiva migliore e da sola sufficiente ad assicurare le minori diffusione e trasmissione delle infezioni sia nosocomiali che occupazionali nell'ambito emodialitico (e ovviamente non solo emodialitico). Abbiamo sottolineato anche l'importanza del monitoraggio continuo e costante delle misure adottate e dei risultati ottenuti, nell'ottica di un miglioramento continuo, come misura non accessoria, bensì necessaria e obbligatoria per la riuscita della prevenzione (9).

Si partiva da un assunto proprio dei CDC ... considerare ogni paziente come potenzialmente infetto e infettante, indipendentemente dalla conoscenza o meno del suo stato effettivo di infezione... (10), che, a nostro parere, banalizza di per sé proprio la misura "drastica" della contumacia. Infatti, la conoscenza dello stato sierologico dei nostri pazienti non è certo in grado di affrancarci dal rischio effettivo di diffusione e trasmissione dei patogeni ematogeni nei nostri centri. Sapere che un paziente "oggi" è sieronegativo non ci rassicura su come lo stesso paziente potrà essere un domani rispetto a quel patogeno, ma anche rispetto ad altri conosciuti o non ancora conosciuti: quante volte abbiamo affermato che un paziente è "negativo e non riveste alcun problema infettivologico" pur sapendo dell'esistenza di patogeni non ancora noti, di periodi finestra particolarmente protratti nei pazienti uremici, dell'oligo-sintomaticità di tali infezioni (specialmente nei pazienti in dialisi) e dell'esistenza di forme occulte (anche se relativamente rare) $\mathrm{HBV}$ e HCV. Perciò, la politica preventiva migliore per la diffusione delle infezioni ematogene risiede nell'adozione completa, continua e da parte di tutti gli operatori di quelle precauzioni sostenibili e realmente applicabili in ogni centro (vecchio o nuovo), che sono le Precauzioni Universali e Speciali $(8,11-13)$.

In modo quantomeno critico, nello stesso articolo, abbiamo posto l'attenzione sul confronto tra l'importanza della contumacia e quella di effettivi protocolli/percorsi di raccomandazione e di preparazione/formazione e aggiornamento costante del personale delle sale dialisi, citando le Linee Guida anglosassoni, che raccomandano in modo esplicito come, in occasione di trattamenti con pazienti infetti, debba essere impiegato criticamente il personale più esperto (14). A questo proposito, sento di dover andare oltre, affermando che tutto il personale, in particolare quello infermieristico, dovrebbe essere sempre e comunque il "più esperto", affermazione che deve essere rivolta in modo particolare a chi ha la gestione amministrativa della sanità.

\section{La posizione dei CDC: perché si e perché no}

Fabrizi nella sua revisione riporta i dati ormai storici (CDC) di un'indagine retrospettiva (dalla fine degli anni ' 70 agli anni '90) di Tokars et al., in cui gli Autori descrivono come l'adozione contemporanea di più misure, ovvero l'implementazione delle precauzioni e la contumaciazione $(15,16)$, sia associata a una riduzione della diffusione dell'infezione HBV nei pazienti e negli staff dei centri dialisi. Per la precisione, l'incidenza di HBsAg nei centri che usavano la contumacia 
si riduceva dal $3.9 \%$ allo $0.2 \%$ /anno, mentre quella nei centri che non la usavano (ma che implementavano le precauzioni) si riduceva dal $6.8 \%$ allo $0.6 \%$ /anno. Pur notando che la $\mathrm{p}$ tra le due percentuali ottenute raggiunge una significatività statistica $(p<0.05)$, deve essere notato che l'azzeramento non veniva raggiunto con nessuno dei due presidi attuati e che il delta percentuale (a causa di una più elevata prevalenza di partenza) risultò maggiore nei centri che non contumaciavano; inoltre, deve essere tenuto presente che si trattava di un'indagine retrospettiva. In sintesi, i dati succitati non permettono di chiarire se il miglioramento delle percentuali fosse attribuibile all'adozione di una politica di isolamento o al rinforzo delle procedure di controllo alias procedure Universali e Speciali. Per perorare ancora la causa della contumacia, Fabrizi cita poi altri studi dei $\mathrm{CDC}$, in cui sono riportate varie cause e concause nel determinismo della diffusione dell'HBV nei centri (Tab. II) $(17,18)$.

Come si può notare dalla Tabella II, le cause citate negli studi non sono univoche, ma quasi sempre "commiste" tra loro e pertanto non possono essere chiaramente conclusive. Malgrado ciò viene riportato di seguito che ... i CDC puntualizzano il concetto che le precauzioni Universali e quelle Specifiche o Speciali potrebbero essere sufficienti per il controllo dell'HBV nei centri dialisi, quando sono seguite in modo rigoroso e reiterato nel tempo ... ma che, a causa dell'elevata concentrazione del virus nel sangue e della sua resistenza nell'ambiente (entrambe molto maggiori rispetto a HCV e HIV, per esempio) ... anche minime violazioni delle procedure di controllo ... possono essere responsabili di focolai epidemici di $H B V$ nei centri dialisi. Questo è il razionale delle procedure di isolamento per i pazienti HBsAg positivi $(3,19)$. Viene riportato anche un dato quantomeno opinabile di un recente studio policentrico prospettico (DOPPS, anno di pubblicazione 2003) (4), ovvero un'associazione fra rischio relativo (RR) di sieroconversione HBV e vaccinazione antiHBV. In realtà, la spiegazione potrebbe essere dovuta proprio al fatto che, nei

\section{TABELLA II - CAUSE DI DIFFUSIONE DELL'INFEZIONE HBV NEI CENTRI DIALISI, RIPORTATE DA STUDI DEI CDC NEGLI ANNI '90}

Gestione di almeno un paziente HBsAg positivo nel centro nell'ultimo mese dell'anno precedente

Episodio di epatite acuta HBV in almeno un paziente nell'anno precedente

Mancato uso di sale e/o macchine dialisi dedicate per soggetti HBsAg positivi

Una presenza di pazienti vaccinati inferiore al 50\%

Mancato rispetto del controllo mensile per HBsAg nei pazienti suscettibili di infezione

Condivisione tra pazienti di farmaci multidose o altro strumentario

Assistenza contemporanea del personale a pazienti positivi e negativi suscettibili di infezione

Eccessiva enfasi per le Precauzioni Universali a discapito di quelle Specifiche

Inadeguato addestramento del personale dei centri dialisi o deficit nel rapporto infermieri/pazienti centri a maggiore incidenza di infezione, viene intrapreso o, comunque, perseguito in modo più serrato il protocollo di vaccinazione. Questo dato, però, deve far riflettere sulla fallacità e sulla difficoltà di far luce sulla reale epidemiologia dell'infezione nei centri dialisi, pur con studi disegnati ad hoc. Un'altra osservazione di quello studio fu l'associazione tra la riduzione del rischio di sieroconversione per HBV e l'uso di protocolli per il controllo della diffusione dell'infezione, senza però che fossero sempre specificate le misure adottate in tali protocolli. In ogni caso, la riduzione del rischio di sieroconversione con la segregazione dei pazienti HBsAg positivi non raggiunse la significatività statistica pur ottenendo una riduzione del RR del 22\%. Neppure l'abilità/training dello staff infermieristico risultò essere un fattore statisticamente significativo per la riduzione del rischio di sieroconversione, ma, alla fine, gli Autori, pur ammettendo di non essere riusciti a dimostrare la significatività statistica tra la riduzione del RR per la sieroconversione HBV e la contumaciazione dei pazienti positivi, riferivano che questa è plausibile da un punto di vista "biologico" e "clinico" e che pertanto non si sentivano confidenti nel rigettare la necessità della segregazione dei pazienti HBV positivi, concludendo che ... The observed variation suggests that further clarification of optimal practice patterns at the facility level may indicate opportunities for improved $H B V$ control... (4).

\section{Altre valutazioni necessarie}

Il punto specifico delle precauzioni supplementari emanato dai CDC che recita che i pazienti positivi e i negativi-protetti possono essere dializzati nello stesso turno, nella stessa stanza e sulle stesse macchine e gestiti dal medesimo personale ha tra le possibili obiezioni (come cita lo stesso Fabrizi nella sua review) la non assoluta protezione fornita dagli anticorpi antiHBs, l'esistenza di ceppi mutanti di HBV in grado di infettare anche pazienti con titolo protettivo di anticorpi antiHBs (specialmente se immunodepressi) $(3,20)$ e la possibilità di infezioni occulte sia HBV che HCV (21-27).

\section{Conclusioni}

Questo contributo vuole essere, alla luce delle attuali conoscenze ed esperienze, una riflessione sull'utilità e sulla sostenibilità della separazione dei pazienti HBsAg positivi nei centri dialisi.

È convinzione di chi scrive che la contumaciazione non possa più essere ritenuta il punto di forza per difendere i pazienti emodializzati nei centri da questa infezione (e da tutte le altre infezioni di origine ematogena), come si è invece ritenuto nei trenta-quaranta anni precedenti. Punti di forza "almeno" altrettanto importanti (e per chi scrive certamente più importanti) sono la vaccinazione di pazienti e personale (viene attuata ancora in modo sufficiente?), la riduzione e la sicurezza delle pratiche emotrasfusionali, ma, soprattutto, l'adozione e la continua implementazione delle precauzioni Universali e Specifiche. In ciò, oltre a quanto scritto sopra, dovremmo farci guidare anche dall'esperienza dell'infezione $\mathrm{HCV}$, di cui siamo riusciti ad azzerare l'incidenza con le sole pratiche 
precauzionali, ma non con la sola contumacia (cioè senza la stretta osservazione delle precauzioni) $(8,28-31)$ e senza fattori confondenti come la vaccinazione. Ci sono poi altri motivi che dovrebbero indurci alla convinzione che le misure principali sono in realtà le precauzioni. Queste infatti devono essere osservate comunque anche nei pazienti separati perché:

- non esiste solo l'infezione HBV;

- ogni paziente (anche quello oggi negativo) è potenzialmente positivo per qualsiasi patogeno;

- i pazienti HBsAg negativi e HBcAb positivi possono tornare (anche se raramente) sieropositivi per l'antigene di superficie e quindi infettanti;

- esistono infezioni HBV (ma anche HCV) occulte.

Tutto ciò dovrebbe renderci confidenti e convinti che la Regola delle $3 T$ (adozione di tutte le precauzioni, Universali e Speciali per la dialisi, da parte di tutti i componenti degli staff dialitici, in tutte le pratiche assistenziali) ci permette una prevenzione della diffusione delle infezioni ematogene, non solo di quella $\mathrm{HBV}$, ma anche di tutte le altre note o non ancora note. Pertanto, ogni membro dello staff ha il dovere di applicare le precauzioni, che ogni centro dovrebbe avere preventivamente stilato e costantemente aggiornato/implementato, poiché ogni paziente ha il diritto di essere assistito nella completa osservanza delle precauzioni. Ritengo che, se queste venissero adottate nella loro completezza, la separazione dei pazienti infettanti da quelli potenzialmente suscettibili di contrarre un'infezione non aggiungerebbe alcun vantaggio. Le precauzioni dovrebbero esser ritenute da tutti il gold standard per ogni paziente di ogni centro dialisi.

In accordo con tali affermazioni è l'esperienza dell'ospedale "Spallanzani" di Roma (9), presso il quale, in un periodo di tempo di 10 anni, con la pedissequa osservanza delle sole (ma di tutte) precauzioni (Universali e Speciali) non si sono avuti casi di sieroconversione nonostante l'elevata pressione infettivologica di quel centro e l'occorrenza di alcuni incidenti ad alto rischio di diffusione ematogena (scoppio di un filtro, per esempio) durante le pratiche assistenziali.

Per quanto riguarda invece la sostenibilità economica (e logistica) delle misure di prevenzione, non c'è la minima ombra di dubbio del vantaggio offerto dalle precauzioni rispetto alla separazione dei pazienti positivi (che non può essere, comunque, disgiunto dall'osservanza delle stesse precauzioni): seguendo la logica della politica di segregazione dei pazienti "positivi", quante aree dovremmo avere (anche in centri che raccolgano pazienti da aree più o meno vaste) e, quindi, quanto personale e strumenti occorrerebbero per poter abbassare o annullare il rischio di trasmissione dei patogeni ematogeni tra i nostri emodializzati? Come già affermato nel lavoro sul GIN del 2009 (8), in un'epoca in cui l'allocazione delle risorse economiche disponibili ha un'importanza crescente, stride che alcuni centri dialisi debbano avere aree separate a disposizione dei pazienti HBV infettanti quando esistono misure che senza questo aggravio organizzativo, e comunque dovute da tutti e per tutti, sono in grado di fare meglio o altrettanto. Oggi non è più sostenibile che il razionale della contumaciazione dei pazienti HBV positivi possa trovare ragione nell'elevata sopravvivenza virale nell'ambiente e nell'elevata carica ematica, quando sappiamo che, grazie all'applicazione delle precauzioni, nessun virus ematogeno potrà trovarsi/restare impunito su superfici, suppellettili, strumentazioni, mani e così via, né potranno avvenire manovre assistenziali errate capaci di diffondere l'infezione (per esempio, preparazione per la somministrazione parenterale che non sia one vial, one patient, no re-entry or re-use).

Ribadendo ancora quanto già asserito nel 2009 (8), la SEPARAZIONE degli HBsAg positivi potrebbe servire solo per alcuni pazienti (i negativi), solo per quel patogeno (in questo caso $\mathrm{HBV}$ ) e, probabilmente, per un tempo che potrebbe anche essere limitato (per esempio, il periodo finestra); invece, le PRECAUZIONI UNIVERSALI E SPECIALI servono per tutti i pazienti (negativi e positivi), per tutti i patogeni (anche quelli non ancora noti) e verosimilmente per sempre, errore umano permettendo, ma questa è una problematica e una sfida che dovranno essere regolate in primis dalle organizzazioni sanitarie, che dovranno impegnarsi sempre più e meglio per la tutela di tutti quanti, pazienti e operatori (32).

\section{Riassunto}

Il presente contributo cerca di analizzare criticamente il ruolo della contumacia in relazione al rischio della trasmissione delle infezioni ematogene in emodialisi ospedaliera. Per fare ciò, l'Autore si avvale del confronto di due articoli apparsi sul GIN, organo ufficiale della SIN. Il primo si fonda sui dati e sulle raccomandazioni emanate dalla maggiore agenzia per la prevenzione delle infezioni (CDC), che, in aggiunta alle note precauzioni Universali e Speciali, raccomanda alcune procedure Supplementari, tra cui l'isolamento dei pazienti HBsAg positivi. Il secondo articolo si fonda sul fatto che la migliore misura preventiva e sufficiente per assicurare le minori diffusione e trasmissione delle infezioni sia nosocomiali che occupazionali risiede nell'adozione costante e continua di tutte le precauzioni Universali e Speciali, sottolineando, al contempo, l'importanza del monitoraggio continuo e costante delle misure adottate e dei risultati ottenuti. Altre possibili obiezioni alla misura dell'isolamento sono la non assoluta protezione fornita dagli anticorpi antiHBs, l'esistenza di ceppi mutanti di HBV in grado di infettare anche pazienti con titolo protettivo di anticorpi antiHBs e la possibilità di infezioni occulte. In un'epoca in cui l'allocazione delle risorse economiche ha un'importanza crescente, stride che i centri dialisi debbano avere aree separate a disposizione dei pazienti HBV infettanti, quando esistono misure che, senza questo aggravio organizzativo, sono in grado di fare meglio o altrettanto. Oggi non è più sostenibile che il razionale della contumaciazione dei pazienti HBV positivi possa trovare la sua maggiore ragione nell'elevata sopravvivenza virale nell'ambiente e nell'elevata carica ematica, quando sappiamo che, grazie all'applicazione delle precauzioni, nessun virus ematogeno potrà/dovrà trovarsi o restare impunito su superfici, suppellettili, strumentazioni, mani e così via.

Parole chiave: Precauzioni Universali e Speciali, Contumacia, HBV e HCV, Prevenzione della diffusione delle infezioni ematogene, Emodialisi 
Dichiarazione di conflitto di interessi: L'Autore dichiara di non avere conflitto di interessi.

Contributi economici agli Autori: L'Autore dichiara di non aver ricevuto sponsorizzazioni economiche per la preparazione dell'articolo.
Indirizzo dell'Autore:

Dr. Marco Lombardi

S.S. Dialisi del Mugello

Via della Resistenza

50032 Borgo S. Lorenzo, Firenze

lombardim@tin.it

\section{Bibliografia}

1. Lombardi M, Cerrai T, Geatti S, et al. Results of a National epidemiological investigation on $\mathrm{HCV}$ infection among dialysis patients. (Survey by the Italian Branch of EDTNA/ERCA). J Nephrol 1999; 12: 322-7.

2. Alavian SM. A shield against a monster: hepatitis $\mathrm{C}$ in hemodialysis patients. World J Gastroenterol 2009; 15 (6): 641-6.

3. Fabrizi F, Martin P, Messa PG. HCV, HBV, HIV ed emodialisi. G Ital Nefrol 2013; 30 (4)

4. Burdick RA, Bragg-Gresham JL, Woods JD, et al. Patterns of hepatitis B prevalence and seroconversion in hemodialysis units from three continents: the DOPPS. Kidney Int 2003; 63 (6): 2222-9.

5. Fissell RB, Bragg-Gresham JL, Woods JD, et al. Patterns of hepatitis $\mathrm{C}$ prevalence and seroconversion in hemodialysis units from three continents: the DOPPS. Kidney Int 2004; 65 (6): 2335-42.

6. Finelli L, Miller JT, Tokars JI, et al. National surveillance of dialysisassociated diseases in the United States, 2002. Semin Dial 2005; 18 (1): 52-61.

7. Tokars JI, Alter MJ, Favero MS, et al. National surveillance of dialysis associated diseases in the United States, 1991. ASAIO J 1993; 39 (4): 966-75.

8. Lombardi M, Cherubini C. La mancata osservanza di tutte le precauzioni speciali è un reale rischio clinico in dialisi. G Ital Nefrol 2009; 26 (5): 625-31.

9. Froio N, Nicastri E, Comandino UV, et al. Contamination by Hepatitis B and C viruses in the dialysis setting. Am J Kidney Dis 2003; 42 (3): 546-50.

10. Centers for Disease Control (CDC). Recommendations for prevention of HIV transmission in health care settings. MMWR Mortal Wkly Rep 1987; 36 (Suppl. 2): S1-18.

11. Lombardi M, Dattolo P, Ferro G, Michelassi S. Prevenzione dell'infezione HCV in emodialisi. G Ital Nefrol 2007; 24 (3): 202-11.

12. Tarchini R, Botti PL, Talassi E, Lambertini D. Ottimizzare spazi e procedure in dialisi. G Ital Nefrol 2000; 17 (1): 44-9.

13. Reccomendations for preventing transmission of infections among chronic haemodialysis patients. MMWR Reccom Rep 2001; 50 (RR-5): $1-43$.

14. http://www.renal.org/pages/pages/guidelines/current/bbv-infection. pho The Renal Association 2009. Guidelines* Blood Borne Virus Infection: Guideline 4.3 - BBV Infection 1C.

15. Centers for Disease Control and Prevention. Hepatitis-control measures for hepatitis B in dialysis centers. Viral Hepatitis Investigations and Control Series 1977.

16. Tokars JI, Alter MJ, Favero MS, et al. National surveillance of dialysis associated diseases in the United States, 1992. ASAIO J
1994; 40 (4): 1020-31

17. Tokars JI, Alter MJ, Miller E, et al. National surveillance of dialysis associated diseases in the United States--1994. ASAIO J 1997; 43 (1): 108-19.

18. Favero M, Alter M. The re-emergence of hepatitis B virus infection in haemodialysis centers. Semin Dial 1996; 9: 373-4.

19. Kellerman S, Alter MJ. Preventing hepatitis B and hepatitis C virus infections in end-stage renal disease patients: back to basics. Hepatology 1999; 29 (1): 291-3.

20. Blaich A, Manz M, Dumoulin A, et al. Reactivation of hepatitis B virus with mutated hepatitis B surface antigen in a liver transplant recipient receiving a graft from an antibody to hepatitis B surface antigen- and antibody to hepatitis B core antigen-positive donor. Transfusion 2012; 52 (9): 1999-2006.

21. Hollinger FB, Habibollahi P, Daneshmand A, Alavian SM. Occult hepatitis $\mathrm{B}$ infection in chronic hemodialysis patients: current concepts and strategy. Hepat Mon 2010; 10 (3): 199-204.

22. Mina P, Georgiadou SP, Rizos C, et al. Prevalence of occult hepatitis B virus infectrion in hemodialysis patients from central Greece. World J Gastroenterol 2010; 16 (2): 225-31.

23. Di Stefano M, Volpe A, Stallone G, et al. Occult HBV infection in hemodialysis settino is marked by presence of isolated antibodies to HBcAg and HCV. J Nephrol 2009; 22: 381-6.

24. Goral V, Ozkul H, Tekes S, et al. Prevalence of occult HBC infection in haemodialysis patients wuith chronic HCV. World J Gastroenterol 2006; 12 (21): 3420-4.

25. Siagris D, Christofidou M, Triga K, et al. Occult hepatitis B virus infection in hemodialysis patients with chronic HCV infection. J Nephrol 2006; 19: 327-33.

26. Barril G, Castello I, Arenas MD, et al. Occult hepatitis C virus infection among hemaolialysis patients. J Am Soc Nephrol 2008; 19: 2288-92.

27. Carreno V. Occult hepatitis $C$ virus infection: a new forn of hepatitis C. World J Gastroenterol 2006; 12 (43): 6922-5.

28. Jadoul M, Cornu C, van Ypersele de Striohu C. Universal precautions prevent hepatitis $\mathrm{C}$ virus transmission: a 54 month follow-up of the Belgian Multicenter Study. The Universitaires Cliniques St-Luc (UCL) Collaborative Group. Kidney Int 1998; 53: 1022-5.

29. Lombardi M, Cerrai T. Infezione HCV in emodialisi: un problema controverso. G Ital Nefrol 1995; 12: 345-6.

30. Lombardi M, Cerrai T. Prevenzione della diffusione dell'HCV in emodialisi. G Ital Nefrol 1996; 13: 419-21.

31. Basile C. Diffusione dell'HCV in emodialisi: isolamento contumaciale versus precauzioni igieniche universali. G Ital Nefrol 2001; 18: 620-1.

32. Catino M. Oltre l'errore umano. Per una teoria organizzativa degli incidenti nelle organizzazioni. G Ital Nefrol 2009; 26: 110-7. 